\title{
Maternal food restriction in the second half of pregnancy affects vascular function but not blood pressure of rat female offspring
}

\author{
Kathleen Holemans ${ }^{1}$, Robert Gerber $^{2}$, Kris Meurrens ${ }^{3}$, Fred De Clerck ${ }^{4}$, Lucilla Poston ${ }^{2}$ \\ and F. André Van Assche ${ }^{1}$ \\ ${ }^{1}$ Department of Obstetrics and Gynecology, Katholieke Universiteit Leuven, 3000 Leuven, Belgium \\ ${ }^{2}$ Department of Obstetrics and Gynaecology, UMDS, St Thomas' Hospital, London SE1 7EH, UK \\ ${ }^{3}$ Proefdierencentrum, Katholieke Universiteit Leuven, 3000 Leuven, Belgium \\ ${ }^{4}$ Department of Cardiovascular and Pulmonary Pharmacology, Janssen Research Foundation, 2340 Beerse, Belgium
}

(Received 27 April 1998 - Revised 8 July 1998 - Accepted 3 August 1998)

\begin{abstract}
Food restriction during pregnancy in rats induces intrauterine growth retardation with consequences persisting into adulthood. In the present study we have investigated the hypothesis that malnutrition in pregnant rats may lead to altered cardiovascular function in adult female offspring. Perinatal growth retardation was induced by a $50 \%$ reduction of normal dietary intake in rats during the second half of pregnancy. Systolic and diastolic blood pressure values and heart rate were recorded in conscious female offspring (100 d old) using a femoral artery probe. No significant differences in heart rate, or in systolic and diastolic blood pressures were recorded between control offspring and offspring of nutritionally deprived rats. In order to ascertain whether cardiovascular variables in the offspring were influenced by lactation, subgroups of offspring from food-restricted dams were fostered with lactating dams fed on a normal diet. Blood pressure and heart rate were also found to be normal in these offspring. The rise in blood pressure associated with NO inhibition was similar in all groups. Isolated resistance artery function was assessed in vitro in offspring (100-120 d old) of a second group of semistarved dams. Small mesenteric arteries from these animals showed reduced endotheliumdependent relaxation (to acetylcholine and bradykinin), but enhanced sensitivity to exogenous NO (sodium nitroprusside). We conclude that food restriction during the second half of pregnancy and/or lactation does not induce hypertension in adult offspring, but may effect subtle changes in vascular function.
\end{abstract}

Food restriction: Intrauterine growth retardation: Vascular function

The concept that some adult diseases may result from in utero 'programming' of the fetus has been the subject of much recent investigation. Early studies from one of our laboratories reported that experimentally induced insulindependent diabetes in pregnant rats was associated with disturbed glucose handling and gestational diabetes in the offspring (Aerts \& Van Assche, 1979; Holemans et al. 1991). Studies in human subjects have since documented that low birth weight, particularly thinness at birth resulting from impaired fetal growth in mid to late gestation, is associated with hypertension (Barker, 1995), coronary heart disease (Phillips et al. 1994) and non-insulin-dependent diabetes (Barker et al. 1993; Leon et al. 1996) in later life. Furthermore, blood pressure in 40-year-old offspring is inversely related with the mothers' intake of animal protein and carbohydrate in late pregnancy (Campbell et al. 1996). Prospective nutritional studies are few and necessarily confined to investigations in animals, but the hypothesis has been strengthened by studies in which rats exposed to malnutrition in utero, either in the form of protein deprivation (Langley \& Jackson, 1994; Langley-Evans et al. 1996a) or of severe food restriction (Woodall et al. 1996), demonstrated significantly raised systolic blood pressure in adulthood.

In the present study we have evaluated a number of cardiovascular variables in adult female rats previously growth retarded in utero. Growth retardation was induced by food restriction ( $50 \%$ normal diet) during the second half of pregnancy (with or without food restriction during lactation). In the first series of experiments heart rate, and

\footnotetext{
Abbreviations: $\mathrm{EC}_{50}$, concentration eliciting half the maximal response; NA, noradrenaline; L-NAME, N $\omega$-nitro-L-arginine methyl ester; O-CR, offspring of control maternal rats; O-FR, offspring of rats food-restricted during pregnancy and lactation; O-FR:CL, offspring of rats food-restricted during pregnancy but suckled by control lactating rats.

* Corresponding author: Dr Kathleen Holemans, fax +32 1634 4205, email Kathleen.Holemans@med.kuleuven.ac.be
} 
systolic and diastolic blood pressures were evaluated in conscious 100-d-old offspring of food-restricted rats and in age-matched controls. In a second series of experiments, constrictor responses and endothelium-dependent dilator function were determined in small mesenteric arteries from the adult offspring (100-120 d old) of food-restricted maternal rats.

\section{Materials and methods}

The entire protocol was reviewed and approved by the local ethics committee for animal procedures (Katholieke Universiteit Leuven, Belgium).

\section{Study 1: heart rate and blood pressure measurements}

Animals. The animals used in this study were 100-120d-old female offspring of control and food-restricted maternal rats (Leuven/pfd, KULeuven Breeding Centre, Leuven, Belgium). The control group consisted of nine female offspring of three pregnant control Wistar rats (three female offspring of each maternal rat). During pregnancy and lactation the rats had free access to a standard nonpurified diet (Trouw, Ghent, Belgium) with an approximate composition of $(\mathrm{g} / \mathrm{kg})$ : 210 protein, 40 fat, 510 carbohydrate, 70 ash, 50 cellulose and 120 water. The foodrestricted group consisted of nine female offspring of three food-restricted maternal rats (three female offspring of each maternal rat). Food-restricted maternal rats received $11 \mathrm{~g} / \mathrm{d}$ of a standard non-purified rat diet from day 11 of pregnancy until the day of delivery (day 23) and $20 \mathrm{~g} / \mathrm{d}$ of the same diet during the entire lactation period. This food restriction scheme ensured that the rats received $50 \%$ of the ad libitum food intake (Aerts et al. 1990). To determine whether in utero events or the lactation period had greater influence on the cardiovascular variables studied, pups from three litters were nursed by their mothers (food restriction during pregnancy and lactation; O-FR). Others (pups from three litters) were cross-fostered immediately after birth on three control dams with free access to a standard non-purified diet (food restriction during pregnancy with control lactation; O-FR:CL). In both subgroups three female offspring from each dam were studied (nine in total).

Only offspring of rats with eight or more fetuses were included in the study. After weaning, only the female offspring of each litter were kept and weighed at 21, 28, $35,49,70$ and $91 \mathrm{~d}$ of postnatal age. To prevent maternal rejection, pups were not weighed at birth. All rats had free access to tap water and a standard non-purified diet for the remainder of the study.

Measurement of blood pressure and heart rate. Rats were transported to the Janssen Research Foundation (Beerse, Belgium) when $91 \mathrm{~d}$ old. After 1 week of acclimatization measurements of blood pressure and heart rate were carried out as previously described (Xhonneux et al. 1990). Briefly, the animals ( $100 \mathrm{~d}$ old) were anaesthetized with diethyl ether and a femoral artery and vein dissected free of surrounding tissue and cannulated. The rats were restrained in Bollman cages and local anaesthesia was induced by administration of lidocaine ( $20 \mathrm{ml} / \mathrm{l}$; Astra, Huizingen, Belgium) to the wound. Heart rate and blood pressure measurements were recorded from the output of a pressure transducer (Janssen Scientific Instruments Division, Beerse, Belgium) in a femoral artery catheter. When the animals were fully awake (approximately 60 min after diethyl ether withdrawal), systolic and diastolic arterial blood pressures and heart rate were recorded continuously for an equilibration period of 60 min (MacLab ${ }^{\circledR}$, AD Instruments PTY Ltd, Castle Hill, New South Wales, Australia), during which systolic and diastolic blood pressures and heart rate reached a stable plateau. Recording was then carried out for a further $60 \mathrm{~min}$ experimental period over which the mean values were calculated for the three variables using a customized computer program (Janssen Scientific Instruments Division). To evaluate the relative contribution of $\mathrm{NO}$ to tonic lowering of blood pressure in each group, $4.63 \mathrm{mmol} \mathrm{N} \omega$ nitro-L-arginine methyl ester (L-NAME; Sigma, Bornem, Belgium) $/ \mathrm{kg}$ was then injected into the venous catheter, and systolic and diastolic arterial blood pressures and heart rate recorded for a further $120 \mathrm{~min}$. The mean systolic and diastolic blood pressures and heart rate were calculated over the $120 \mathrm{~min}$ period. Blood pressure and heart rate were measured on three consecutive days in nine animals simultaneously. The rats were used in a randomized manner.

\section{Study 2: vascular function in isolated arteries}

Animals. Female offspring ( $n$ 11) between 100 and $120 \mathrm{~d}$ of age of a second group $(n 6)$ of food-restricted maternal rats (O-FR) were studied for assessment of vascular function. Comparison was made with arteries isolated from 100-120-d-old female offspring ( $n 11)$ of six control maternal rats (O-CR).

Assessment of vascular function. Small mesenteric arteries were mounted as previously described (Mulvany \& Halpern, 1977) on a small vessel wire myograph. Arteries were bathed in physiological saline solution $(\mathrm{mmol} / \mathrm{l}: \mathrm{NaCl}$ 119, $\mathrm{KCl} 4 \cdot 7, \mathrm{CaCl}_{2} 2 \cdot 5, \mathrm{MgSO}_{4}$ 1.17, $\mathrm{NaHCO}_{3} 25$, $\mathrm{KH}_{2} \mathrm{PO}_{4} 1 \cdot 16$, EDTA 0.026 , glucose 6.0) at $37^{\circ}$ and gassed with $\mathrm{CO}_{2}-\mathrm{O}_{2}(5: 95, \mathrm{v} / \mathrm{v})$. The passive tensioninternal circumference characteristics of the arteries were determined by stretching to achieve an internal circumference equivalent to $90 \%$ of that which would be attained when relaxed in situ under a transmural pressure of $100 \mathrm{mmHg}$. To confirm viability of the arteries five contractions ( $2 \mathrm{~min}$ duration) were performed to $5 \mu \mathrm{mol} / \mathrm{l}$ noradrenaline (NA; Winthrop, Guildford, Surrey, UK), a solution of $125 \mathrm{mmol} / \mathrm{l} \mathrm{KCl}$ in physiological saline solution, or a combination of both. Arteries failing to produce active tension equivalent to $100 \mathrm{mmHg}$ were rejected. A cumulative concentration response to NA $\left(10^{-9}-10^{-5} \mathrm{~mol} / \mathrm{l}\right)$ was constructed and arteries then preconstricted with NA at a concentration $(3-5 \mu \mathrm{molNA} / \mathrm{l})$ required to give a submaximal constrictor response. Vasodilator responses to $10^{-9}-10^{-5} \mathrm{~mol} / \mathrm{l}$ acetylcholine (Sigma, Poole, Dorset, UK) were determined with additions at 2 min intervals, and after further preconstrictions to NA, responses to $10^{-9}$ $10^{-5} \mathrm{~mol} / 1$ bradykinin (Sigma) and $10^{-9}-10^{-5} \mathrm{~mol} / 1$ sodium nitroprusside (Sigma) were evaluated. In most experiments, two arteries from each rat were investigated 
simultaneously and the results expressed as the mean of the data pair.

Biochemical analyses. Tail blood samples for measurement of plasma glucose and insulin concentrations were obtained from the dams at 20 d gestation. Tail blood samples were also taken from the offspring at $80 \mathrm{~d}$ of age after an overnight fast for determination of glucose, insulin, triacylglycerols and cholesterol. Plasma glucose was determined with a glucose analyser 2300STAT (Yellow Springs Instruments, Yellow Springs, OH, USA), plasma insulin by radioimmunoassay using rat insulin as a standard, plasma triacylglycerols (Triglycerides GPO-PAP) and cholesterol (Cholesterol CHOL-PAP) with kits from Boehringer (Mannheim, Germany).

\section{Statistical analysis}

Heart rate and blood pressure measurement. Data for blood pressure, heart rate and body weight are given as means with their standard errors (SEM). Statistical analyses for heart rate and blood pressure were carried out using a paired $t$ test for sequential comparisons in the same animals or ANOVA for multiple comparisons followed by an unpaired $t$ test, as appropriate, to assess differences within or between the groups. Two-tailed probabilities $P<0.05$ were considered significant. Growth curves were analysed by ANOVA for multiple comparison followed by a $t$ test ('Statistica'; Statsoft, Tulsa, OK, USA).

Vascular function in isolated arteries. All values are given as means with their standard errors. Tension was calculated as milliNewtons $(\mathrm{mN}) / \mathrm{mm}$ artery length. To account for variation in artery diameter, concentration responses to NA were expressed as a percentage of the contractile response to a depolarizing $\mathrm{K}^{+}$buffer $(124 \mathrm{mmol} / \mathrm{l}$ $\mathrm{K}^{+}$-substituted physiological saline solution). Relaxation to acetylcholine, bradykinin and sodium nitroprusside was expressed as a percentage of the initial precontraction to NA. The concentration (mol/l) which elicited half the maximal response $\left(\mathrm{EC}_{50}\right)$, was calculated for each concentration response curve. Values are given as the $\mathrm{pEC}_{50}$, the negative $\log$ of $\mathrm{EC}_{50}$. When calculation of the $\mathrm{pEC}_{50}$ was not appropriate, responses to vasodilators were compared by the maximum responses to the agonist and curves were compared by summary score (Matthews et al. 1990). Summary values are calculated from individual data points as the sum of responses in each curve divided by the number of concentrations tested. Unpaired Student's two-tailed $t$ test or the Mann-Whitney $U$ test was used for comparison of parametric and non-parametric data respectively (both by 'Instat'; GraphPad Software Inc., San Diego, CA, USA). Significance was assumed if $P<0.05$.

\section{Results}

\section{Study 1: heart rate and blood pressure measurement}

Litter size and mortality. Litter sizes were not significantly altered by food restriction of the maternal rat (pups per litter: controls 10.14 (SE 0.14); food restricted, 10.0 (SE $0.62)$ ). Pup mortality rates per litter in O-FR and O-CR groups were not significantly different (4.6 (SE 4.6) v. 1.4 (SE 1.4$) \% ; P=0.465)$.
Growth rates in offspring of food-restricted rats (Fig. 1). Body weight was significantly reduced in weaned (21-d-old) female O-FR compared with O-CR $(P<0.0001)$. The growth rate subsequently paralleled that of O-CR, but body weight at each point of measurement remained significantly lower $(P<0.0001)$. Newborns of food-restricted rats, fostered by a mother on normal dietary intake (O-FR:CL) weighed more at $21 \mathrm{~d}$ than O-FR $(P<0.0001)$ but weights were lower than those of $\mathrm{O}-\mathrm{CR}$ $(P<0.01)$. Although early growth rates paralleled those of controls, weights remained significantly lower in O-FR:CL at $91 \mathrm{~d}(P<0.001)$.

Systolic and diastolic blood pressures and heart rate (Fig. 2). During the first $60 \mathrm{~min}$ of recording after the equilibration period, heart rate and systolic and diastolic blood pressures were similar for the three groups studied. After administration of L-NAME, heart rate decreased significantly when compared with the value just before administration, and systolic and diastolic blood pressures increased significantly in all groups studied. There were no differences in the mean responses to L-NAME, nor in the maximal effect of L-NAME between groups.

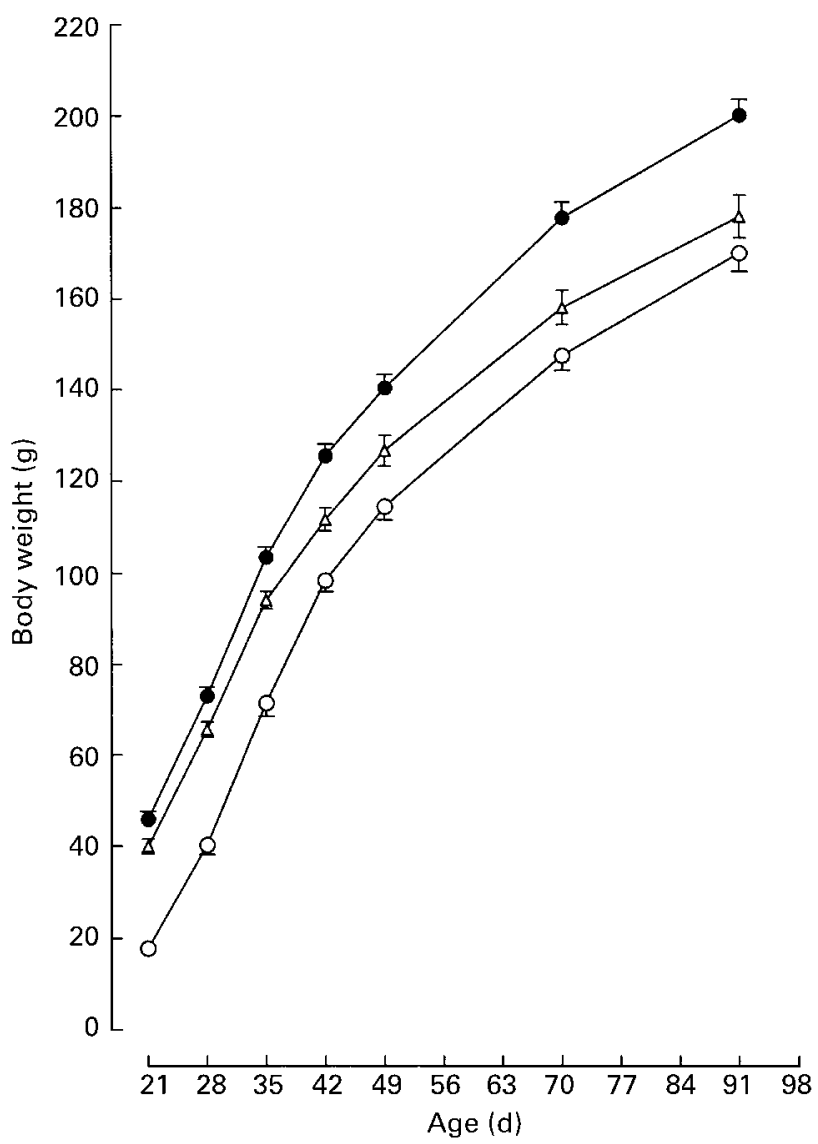

Fig. 1. Postnatal growth curves in female offspring of control rats $(\bullet)$, female offspring of rats food-restricted during pregnancy and lactation $(\bigcirc)$ and female offspring of rats food-restricted during pregnancy but not lactation $(\triangle)$. Values are means with their standard errors indicated by vertical bars for nine rats per group. For details of procedures, see p. 74. 

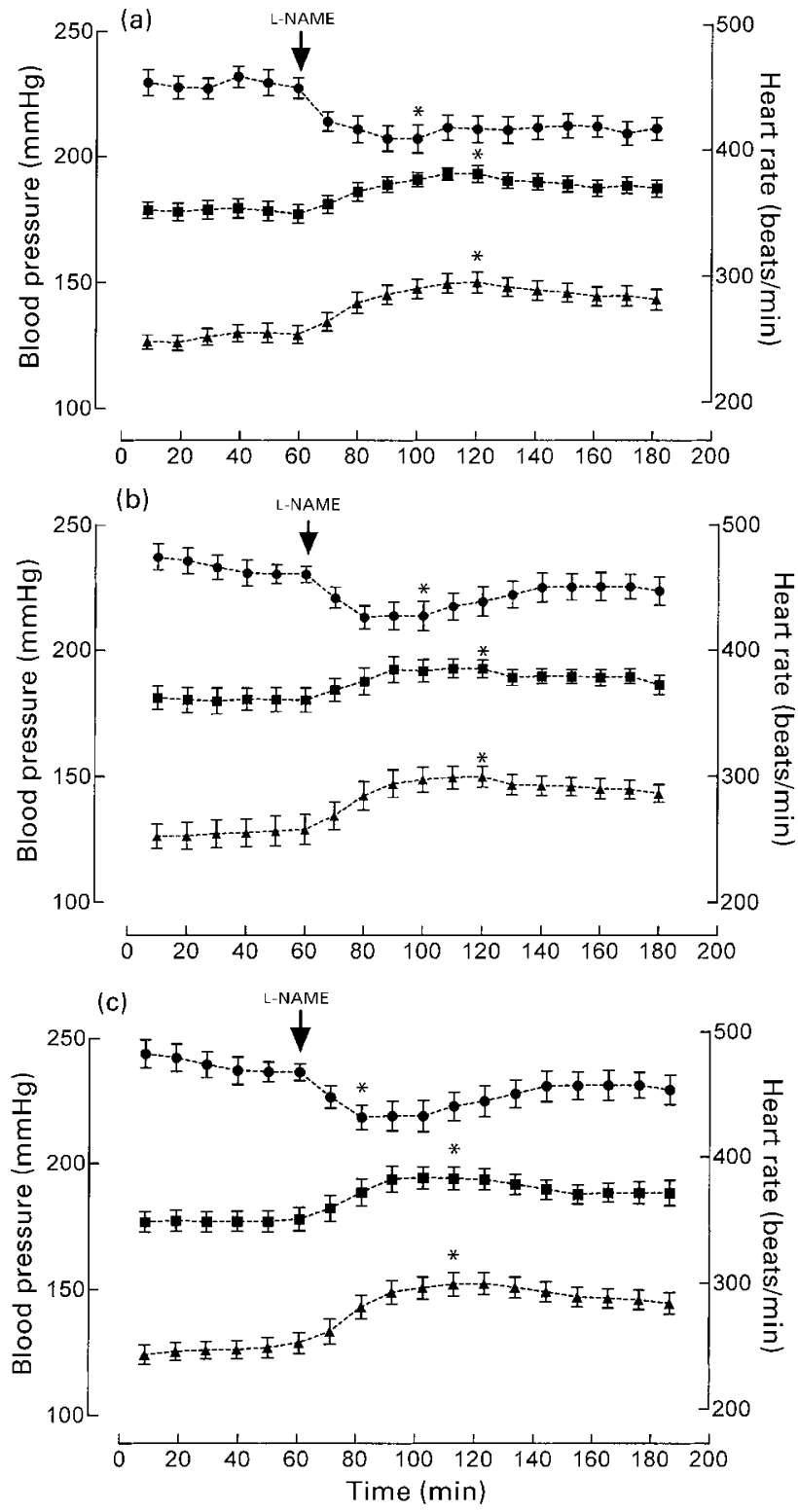

Fig. 2. Systolic ( $\mathbf{\square})$ and diastolic $(\boldsymbol{\Lambda})$ blood pressures and heart rate ()) over a 180 min experimental period in (a) offspring of control rats, (b) offspring of food-restricted rats and (c) offspring of food-restricted rats suckled by control rats. For details of procedures, see p. 74. Values are means for nine rats, with their standard errors represented by vertical bars. ( $\downarrow$ ) Intravenous administration of $\mathrm{N} \omega$-nitro-L-arginine methyl ester (L-NAME). Mean values were significantly different from basal values, ${ }^{*} P<0.05$.

\section{Study 2: vascular function in isolated arteries}

General features. The serum glucose and insulin concentrations were lower in food-restricted maternal rats when compared with control pregnant rats (Table 1). The 80-d-old O-FR had lower body weights than O-CR, $P<0.0001$ (Table 2). Glucose concentrations were higher in O-FR than in $\mathrm{O}-\mathrm{CR}$, whereas insulin concentrations were lower than in O-CR $(P<0 \cdot 05)$. Fasting plasma triacylglycerol and cholesterol concentrations were normal in O-FR (Table 2).

Vascular function. Mean vessel diameters of the arteries from O-CR and O-FR were similar (303 (SE
Table 1. Study 2. Body weight and non-fasting plasma glucose and insulin concentrations in control and food-restricted maternal rats on day 20 of gestation

(Mean values with their standard errors for six rats per group)

\begin{tabular}{|c|c|c|c|c|}
\hline & \multicolumn{2}{|c|}{ Control } & \multicolumn{2}{|c|}{ Food-restricted } \\
\hline & Mean & SEM & Mean & SEM \\
\hline Body weight (g) & 303 & 2 & $248^{\star \star \star}$ & 3 \\
\hline Plasma glucose $(\mathrm{mmol} / \mathrm{l})$ & 5.33 & $0 \cdot 13$ & $4 \cdot 17^{\star *}$ & $0 \cdot 11$ \\
\hline Plasma insulin (nmol/l) & $0 \cdot 16$ & 0.03 & $0.03^{* *}$ & 0.01 \\
\hline
\end{tabular}

7) $\mu \mathrm{m}, n 22$ and 276 (SE 11) $\mu \mathrm{m}, n 22$ respectively). Maximum responses to $125 \mathrm{mmol} / \mathrm{l} \mathrm{K}$-buffer were significantly reduced in the O-FR compared with O-CR (4.01 (SE $0.20)$ v. $4.72($ SE 0.27$) \mathrm{mN} / \mathrm{mm} ; P<0.05)$. There was no significant difference in the constrictor response to NA between $\mathrm{O}-\mathrm{CR}$ and O-FR either in sensitivity $\left(\mathrm{pEC}_{50}: 5.63\right.$ (SE 0.06) in O-CR v. 5.72 (SE 0.05) in O-FR, $P=0.293$ ) or in maximal response (97.85 (SE 1.90)\% of maximum constriction to $\mathrm{K}^{+}$v. 102.53 (SE 2.80)\%, $P=0.170$ ). Maximal relaxation to the endothelium-dependent vasodilators, acetylcholine and bradykinin were significantly reduced in O-FR (acetylcholine 87.7 (SE 2.65) v. 95.51 (SE 1.36 ) \% in O-CR, $P<0.05$; bradykinin 29.93 (SE 3.15) $v$. 40.97 (SE 2.80) \% in O-CR, $P<0.05$ ) (Fig. 3(a and b)). In contrast, relaxation to the NO donor, sodium nitroprusside, was enhanced $(P<0.01$, by summary score $)$ in O-FR (Fig. 3(c)).

\section{Discussion}

In this study we have shown that perinatal growth retardation in rats, induced by a $50 \%$ restriction of food to the maternal rate during the second half of pregnancy, when fetal growth is maximal, is not associated with alterations in blood pressure or heart rate in adulthood $(100 \mathrm{~d})$. This would contrast, apparently, with a study by Woodall et al. (1996) which has shown a small but significant increase in systolic blood pressure $(5-8 \mathrm{mmHg})$ in much older (30-week-old) offspring of severely dietary restricted rats $(70 \%$ restriction). The study differs from the present investigation as dietary restriction was imposed on the maternal rats from day 1 of pregnancy (Woodall et al. 1996), whereas food restriction was started on day 11 in the present study. The degree of food reduction or an age-related development of raised blood pressure could potentially explain the difference in results, and the findings might also suggest that dietary restriction in early pregnancy could be an important determinant of blood pressure in the offspring. The latter proposal is not supported by a study (Langley-Evans et al. 1996c) which observed no significant effect of maternal protein restriction in the first week of pregnancy on blood pressure of 7-week-old female offspring, and only a minor, but significant elevation in males. However, to our knowledge, no study has similarly evaluated the effect of reduction of all components of the diet in early pregnancy on the blood pressure of offspring of a similar age to those we have studied. 
Table 2. Study 2. Body weight and fasting plasma glucose, insulin, triacylglycerol and cholesterol concentrations in 80-d-old female offspring of control maternal rats $(\mathrm{O}-\mathrm{CR})$ and of food-restricted maternal rats (O-FR)

(Mean values with their standard errors for eleven rats per group)

\begin{tabular}{|c|c|c|c|c|}
\hline & \multicolumn{2}{|c|}{$\mathrm{O}-\mathrm{CR}$} & \multicolumn{2}{|c|}{ O-FR } \\
\hline & Mean & SEM & Mean & SEM \\
\hline Body weight (g) & 179 & 4 & $134^{\star * *}$ & 1 \\
\hline Plasma glucose $(\mathrm{mmol} / \mathrm{l})$ & $6 \cdot 41$ & $0 \cdot 18$ & $6.92^{*}$ & $0 \cdot 12$ \\
\hline Plasma insulin $(\mathrm{nmol} / \mathrm{l})$ & $0 \cdot 10$ & 0.01 & $0.04^{* *}$ & 0.01 \\
\hline Plasma triacylglycerols $(\mathrm{mmol} / \mathrm{l})$ & 0.61 & 0.04 & 0.72 & 0.04 \\
\hline Plasma cholesterol $(\mathrm{mmol} / \mathrm{l})$ & 1.73 & 0.08 & 1.68 & 0.04 \\
\hline
\end{tabular}

Mean values were significantly different from control: ${ }^{\star} P<0.05$, ${ }^{\star *} P<0.01$, ${ }^{\star \star \star} P<0.0001$.

In contrast to the small rise in blood pressure observed by Woodall et al. (1996) and the lack of rise in blood pressure in the offspring of dietary deprived rats in the present study, protein restriction (casein 60, 90 and $120 \mathrm{~g} / \mathrm{kg}$ diet) throughout rat pregnancy results in a very significant increase in systolic blood pressure in 9-21-week-old offspring (Langley \& Jackson, 1994). Our results may indicate, therefore, that balanced food restriction during pregnancy has a lesser effect on blood pressure in the offspring than the restriction of specific nutrients. Further studies of dietary deprivation of all constituents throughout pregnancy, and in early pregnancy alone, would determine the relative roles of protein and balanced dietary deprivation on offspring blood pressure.

Theoretically, the relatively small numbers of offspring investigated in the present study might prevent detection of small differences in blood pressure between the groups, but in contrast to the measurement of blood pressure with a tail cuff, as used in most similar studies, the variability in the measurement of the blood pressure using a femoral artery transducer was very small and renders this an unlikely explanation for the lack of difference observed.

The present study, in which blood pressure was evaluated by direct measurement from a femoral artery catheter is, to our knowledge, the first to report direct recording of arterial blood pressure from the offspring of nutritionally deprived rats. Whereas similar blood pressures in control rats have been reported previously (Janssen et al. 1989), the blood pressure was higher in our controls than that reported in some studies using the tail cuff method (Langley \& Jackson, 1994; Langley-Evans et al. 1996a) and others with indwelling transducers (Schiffers et al. 1994). There is no obvious explanation for the high degree of variability between studies other than differences in the method of measurement and the use of different strains. The use of female rats alone, which were used in the present study in order to compare with earlier similar investigations from our laboratory, is not a likely factor since previous studies have suggested there is no sex difference (Langley-Evans et al. 1996b).

To avoid maternal rejection, birth weights of the newborn pups from the three groups of offspring were not determined. However, we have previously shown body weight to be $20 \%$ lower in 22-d-old fetuses of similarly foodrestricted rats (Holemans et al. 1997b). Growth retardation was also clearly indicated by the postnatal growth patterns which showed that food restriction during pregnancy (with or without food restriction during lactation) had long-lasting effects on body weight of the adult offspring. It is unlikely that the reduced body weight in O-FR was the result of reduced food intake during growth as we have previously shown that their food intake (relative to body weight) is not different from that of controls (Holemans et al. 1997b). It is of interest that the growth curves for the offspring indicated that dietary deprivation during pregnancy alone was
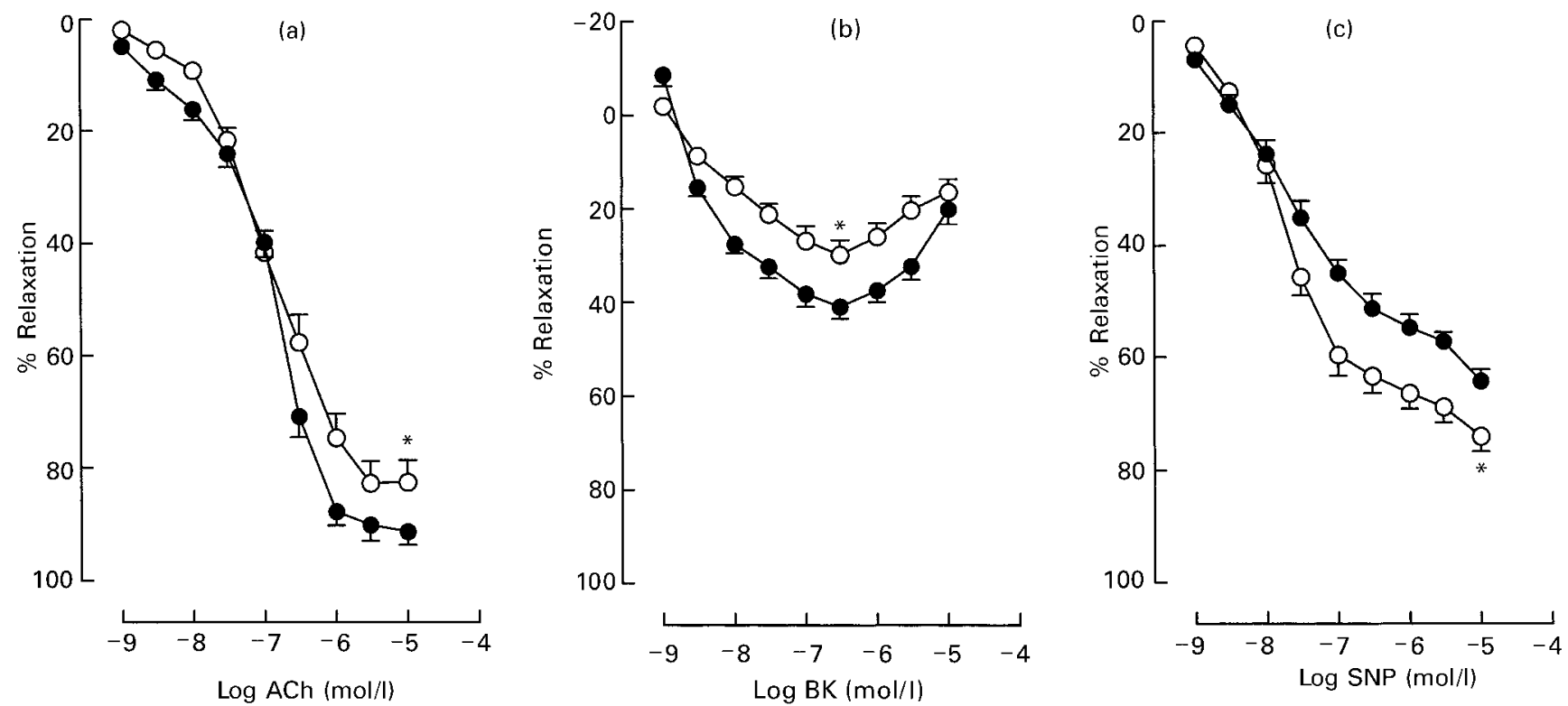

Fig. 3. Concentration-dependent relaxation to (a) acetylcholine (ACh), (b) bradykinin (BK) and (c) sodium nitroprusside (SNP) in mesenteric small arteries preconstricted with submaximal noradrenaline $(3-5 \mu \mathrm{mol} / \mathrm{l})$ from offspring of control rats $(\bullet)$ and offspring of food-restricted dams $(O)$. Tension is given as percentage relaxation of the initial response to noradrenaline. Values are means for ten (control, SNP) or eleven rats, with their standard errors represented by vertical bars. ${ }^{*}$ For $\mathrm{ACh}$ and BK, values for the two groups were significantly different $(P<0.05)$ by maximal relaxation and summary scores. For SNP, values for the two groups were significantly different $(P<0.01)$ by summary scores. 
sufficient to induce a long-term effect on body weight. Indeed, in a recent study we have shown that the female offspring of rats nutritionally deprived (food restriction or low protein) during lactation alone demonstrated 'catch-up' of body weight (Holemans et al. 1997a).

Despite normal blood pressures and normal heart rate, we chose to investigate vascular function in the offspring of food-restricted rats suckled by their own dams since previous studies from our laboratory have indicated abnormalities of glucose homeostasis in these animals (Holemans et al. 1996, 1997b), which could affect vascular function. Subtle changes were recorded in the small mesenteric arteries investigated. The difference in the maximal response to a depolarizing $\mathrm{K}^{+}$solution is likely to reflect smaller vascular smooth-muscle mass in growth-retarded offspring and the normal constriction to NA (once corrected for the differences in $\mathrm{K}$ contraction), did not suggest a disorder of constrictor function. Small, but significant, reductions were observed in the dilator responses to acetylcholine and bradykinin, indicative of a reduction in synthesis of the endothelium-dependent vasodilator, $\mathrm{NO}$ or a decrease in half-life as both agents evoke vasodilatation, at least in part, through NO release. It is relevant that previous studies in the same vascular bed of spontaneously hypertensive rats (Clozel et al. 1990; Luscher et al. 1992) have shown abnormalities of vascular endothelial dilator function that have been implicated in the evolution of raised blood pressure. In this study relaxation to sodium nitroprusside, which evaluates responsiveness of the vascular smooth muscle to exogenous NO, was enhanced. Theoretically, this could reflect a compensatory response to tonic NO depletion. We suggest that one abnormality offsets the other; the reduction in NO synthesis or availability being paralleled by increased sensitivity, so resulting in no net change of NO-induced vasodilatation, and thus no effect on blood pressure. These observations in vitro would accord with those observed after infusion of L-NAME in vivo, which showed a similar enhancement of blood pressure in all groups.

To our knowledge only one study has investigated the possibility that growth restriction in human pregnancy may be associated with vascular dysfunction and, in agreement with the present investigation, documented an apparent effect of growth restriction on endothelial function. Leeson et al. (1997) used a non-invasive method to evaluate endothelium-dependent dilatation in the brachial artery of children. The authors reported a significant, graded and positive association of birth weight with flow-mediated dilatation, whilst other childhood cardiovascular risk factors were unrelated to flow-induced response.

The mechanism underlying the reduced capacity to synthesize NO is nonetheless of interest as it suggests a defect of endothelial function. There is scant information regarding the effects of dietary deprivation on vascular function whereas dietary excess, in the form of a high lipid intake, has frequently been implicated in endothelial dysfunction. Possible candidates leading to endothelial malfunction in the offspring of the food-restricted animals include the mild degree of hyperglycaemia observed, as we and others (for reviews see Poston \& Taylor, 1995; Tribe \& Poston, 1996) have implicated hyperglycaemia in endothelial dysfunction associated with insulin-dependent and non-insulin-dependent diabetes mellitus. Hyperglycaemia, through a wide spectrum of biochemical pathways, may reduce endothelial synthesis of NO. The hypoinsulinaemia observed could also blunt NO synthesis as, in some vascular beds, insulin stimulates NO synthase (EC 1.14.13.39) (Chen \& Messina, 1996). Recent studies in the offspring of foodrestricted rats showed that the primary defect in glucose homeostasis appears to be at the endocrine pancreas (Garofano et al. 1997). Additionally, O-FR are also insulin resistant (Holemans et al. 1996). In contrast to the insulinresistant patient, insulin resistance is attributable to decreased responsiveness of the liver to insulin, and normal peripheral glucose utilization (Holemans et al. 1996, 1997b). Thus, those earlier studies, together with the present investigation, lend some experimental support to retrospective epidemiological evidence proposing that low birth weight is associated with non-insulin-dependent diabetes in later life (Barker et al. 1993).

To our knowledge few studies have investigated the effect of dietary manipulation in pregnancy on vascular function in the offspring. It is of relevance that in two recent studies we have shown a very pronounced blunting of the response to acetylcholine in the neonatal vasculature from offspring of streptozotocin-diabetic rats on a high-fat diet (Koukkou et al. 1997) and in the adult offspring of streptozotocin-diabetic rats (Holemans et al. 1998). The abnormalities described in the present study and in our recent work suggest that in utero events may have lasting consequences in terms of in vitro vascular function. Whether these may contribute to overt adult disease remains to be determined. Further studies are indicated in which dietary restriction is imposed throughout pregnancy and at different stages in pregnancy, and in which the offspring are studied from birth to old age. More detailed evaluation of vascular function in a range of vascular beds would provide insight into the defects observed in this study and the likelihood of their contribution to overt vascular disease.

In conclusion, this study does not support the hypothesis that malnutrition of the fetus in utero may contribute to hypertension in adulthood. The modest disorders of vascular function described would tend to offset one another. Nonetheless, the demonstration that in utero events can alter vascular function opens an exciting avenue of research, not only in relation to undernutrition, but to a wide range of dietary extremes and pathological disorders of pregnancy that may affect the fetus.

\section{Acknowledgements}

We wish to thank Rita De Reese (Janssen Research Foundation) and Rieta van Bree (Department of Obstetrics and Gynecology, Katholieke Universiteit Leuven) for technical assistance, and Guido Pauwels for taking care of the animals. The authors acknowledge the kind support from Tommy's Campaign and the Trustees of St Thomas' Hospital for supporting this study.

\section{References}

Aerts L, Holemans K \& Van Assche FA (1990) Maternal diabetes during pregnancy: consequences for the offspring. Diabetes and Metabolism Reviews 6, 147-167. 
Aerts L \& Van Assche FA (1979) Is gestational diabetes an acquired condition? Journal of Developmental Physiology 1, 219-225.

Barker DJ (1995) Fetal origins of coronary heart disease. British Medical Journal 311, 171-174.

Barker DJ, Hales CN, Fall CH, Osmond C, Phipps K \& Clark PM (1993) Type 2 (non-insulin-dependent) diabetes mellitus, hypertension and hyperlipidaemia (syndrome $\mathrm{X}$ ): relation to reduced fetal growth. Diabetologia 36, 62-67.

Campbell DM, Hall MH, Barker DJP, Cross J, Shiell AW \& Godfrey KM (1996) Diet in pregnancy and the offspring's blood pressure 40 years later. British Journal of Obstetrics and Gynaecology 103, 273-280.

Chen YL \& Messina EJ (1996) Dilation of isolated skeletal muscle arterioles by insulin is endothelium dependent and nitric oxide mediated. American Journal of Physiology 270, H2120-H2124.

Clozel M, Kuhn H \& Hefti F (1990) Effects of angiotensin converting enzyme inhibitors and of hydralazine on endothelial function in hypertensive rats. Hypertension 16, 532-540.

Garofano A, Czernichow P \& Bréant B (1997) In utero undernutrition impairs rat beta-cell development. Diabetologia 10, 1231-1234.

Holemans K, Aerts L \& Van Assche FA (1991) Evidence for an insulin resistance in the adult offspring of pregnant streptozotocindiabetic rats. Diabetologia 34, 81-85.

Holemans K, Gerber RT, Van Assche FA \& Poston L (1998) Adult offspring from diabetic Wistar rats show abnormal endotheliumdependent relaxation and reduced heart rate. Journal of Vascular Research 35, Suppl. 1, 6 Abstr.

Holemans K, Spiessens C, Meurrens K \& Van Assche FA (1997a) Growth patterns after growth retardation in the rat: effect of pregnancy and lactation. American Journal of Obstetrics and Gynecology 176, S128 Abstr.

Holemans K, Van Bree R, Verhaeghe J, Meurrens K \& Van Assche FA (1997b) Maternal semistarvation and streptozotocindiabetes in rats have different effects on the in vivo glucose uptake by peripheral tissues in their female adult offspring. Journal of Nutrition 127, 1371-1376.

Holemans K, Verhaeghe J, Dequeker J \& Van Assche FA (1996) Insulin sensitivity in adult female offspring of rats subjected to malnutrition during the perinatal period. Journal of the Society for Gynecological Investigations 3, 71-77.

Janssen WJ, Van de Water A, Xhonneux R, Reneman RS, Van Neuten JM \& Janssen PAJ (1989) Nebivolol is devoid of intrinsic sympathomimetic activity. European Journal of Pharmacology 159, 89-95.

Koukkou E, Lowy C \& Poston L (1997) The offspring of diabetic rats fed a high saturated fat diet demonstrate abnormal vascular function. Journal of the Society for Gynecological Investigations 4, 115A Abstr.

Langley SC \& Jackson AA (1994) Increased systolic blood pressure in adult rats induced by fetal exposure to maternal low protein diets. Clinical Science 86, 217-222.
Langley-Evans SC, Gardner DS \& Jackson AA (1996a) Association of disproportionate growth of fetal rats in late gestation with raised systolic blood pressure in later life. Journal of Reproduction and Fertility 106, 307-312.

Langley-Evans SC, Gardner DS \& Jackson AA (1996b) Maternal protein restriction influences the programming of the rat hypothalamic pituitary adrenal axis. Journal of Nutrition 126, $1578-1585$.

Langley-Evans SC, Welham JM, Sherman RC \& Jackson AA (1996c) Weanling rats exposed to maternal low-protein diets during discrete periods of gestation exhibit differing severity of hypertension. Clinical Science 91, 607-615.

Leeson CPM, Whincup PH, Cook DG, Donald AE, Papacosta O, Lucas A \& Deanfield JE (1997) Flow-mediated dilatation in 9-11 year old children: the influence of intrauterine and childhood factors. Circulation 96, 2233-2238.

Leon DA, Koupilova I, Lithell HO, Berglund L, Hohsen R, Vagero D, Lithell UD \& McKeigue PM (1996) Failure to realise growth potential in utero and adult obesity in relation to blood pressure in 50 year old Swedish men. British Medical Journal 312, 401-406.

Luscher TF, Boulanger CM, Dohi Y \& Tschudi M (1992) Endothelium dependent regulation of resistance arteries: alterations with aging and hypertension. Journal of Cardiovascular Pharmacology 19, S34-S42.

Matthews J, Altman D \& Campbell M (1990) Analysis of serial measurements in medical research. British Medical Journal 300, 230-235.

Mulvany MJ \& Halpern W (1977) Contractile properties of small arterial resistance arteries in spontaneously hypertensive rats and normotensive rats. Circulation Research 41, 19-26.

Phillips DI, Barker DJ, Hales CN, Hirst S \& Osmond C (1994) Thinness at birth and insulin resistance in adult life. Diabetologia 37, 150-154.

Poston L \& Taylor PD (1995) Endothelial-mediated vascular function in insulin-dependent diabetes mellitus. Clinical Science 88, 245-255.

Schiffers PMH, Fazzi GE, Janssen GMJ, Uitendaal MP, Struijker Boudier HAJ \& DeMey JGR (1994) DNA synthesis in isolated arteries of normotensive and hypertensive rats: effects of the endothelium. Journal of Hypertension 12, 245-250.

Tribe RM \& Poston L (1996) Oxidative stress and lipids in diabetes: a role in endothelium vasodilator dysfunction. Vascular Medicine 1, 195-206.

Woodall SM, Johnston BM, Breier BH \& Gluckman PD (1996) Chronic maternal undernutrition in the rat leads to delayed postnatal growth and elevated blood pressure of offspring. Pediatric Research 40, 438-443.

Xhonneux R, Wouters L, Reneman RS \& Janssen PAJ (1990) The L-enantiomer of nebivolol potentiates the blood pressure lowering effect of the D-enantiomer. European Journal of Pharmacology 181, 261-265. 


\section{Yifingn \\ Access full-text articles from the British Journal of Nutrition on the Internet}

\section{http://nutrition.cabweb.org}

The British Journal of Nutrition is now available on the Internet through Nutrition CABWeb, our new service providing desktop access to information in nutritional science.

The service is open to all until the end of 1998. Access controls will be in place from January 1999, and members of institutions/organizations with a current print subscription will be able to access the electronic version of the $B J N$ for FREE once their institution/organization has registered.

Visit the site now to find out more about the service and for details on how to register.

(10) $\mathrm{CABI}$ Publishing

CAB International

Wallingford

Oxon, OX10 8DE, UK

Tel: +44 (0)1491832111

Fax: +44 (0)1491829198

Email: publishing@cabi.org
(10) CABI Publishing

CAB International

10 East 40th Street, Suite 3203

New York, NY 10016, USA

Tel: +12124817018

Fax: +12126867993

Email: cabi-nao@cabi.org 